\title{
A Family of the Exponential Attractors and the Inertial Manifolds for a Class of Generalized Kirchhoff Equations
}

\author{
Guoguang Lin, Lujiao Yang \\ Department of Mathematics, Yunnan University, Kunming, China \\ Email: gglin@ynu.edu.cn, lj112968y@163.com
}

How to cite this paper: Lin, G.G. and Yang, L.J. (2021) A Family of the Exponential Attractors and the Inertial Manifolds for a Class of Generalized Kirchhoff Equations. Journal of Applied Mathematics and Physics, 9, 2399-2413.

https://doi.org/10.4236/jamp.2021.910152

Received: August 27, 2021

Accepted: September 27, 2021

Published: September 30, 2021

Copyright $\odot 2021$ by author(s) and Scientific Research Publishing Inc. This work is licensed under the Creative Commons Attribution International License (CC BY 4.0).

http://creativecommons.org/licenses/by/4.0/

\begin{abstract}
In this paper, we studied a family of the exponential attractors and the inertial manifolds for a class of generalized Kirchhoff-type equations with strong dissipation term. After making appropriate assumptions for Kirchhoff stress term and nonlinear term, the existence of exponential attractor is obtained by proving the discrete squeezing property of the equation, then according to Hadamard's graph transformation method, the spectral interval condition is proved to be true, therefore, the existence of a family of the inertial manifolds for the equation is obtained.
\end{abstract}

\section{Keywords}

Kirchhoff-Type Equation, Spectral Interval Condition, A Family of the Exponential Attractors, A Family of the Inertial Manifolds

\section{Introduction}

In the study of dynamic behavior for a long time in infinite dimensional dynamical system, the exponential attractors and inertial manifolds play a very important role. In 1994, Foias [1] puts forward the concept of exponential attractor, it is a positive invariant compact set which has finite fractal dimension and attracts solution orbits at an exponential rate. Inertial manifold is finite dimensional invariant smooth manifolds that contain the global attractor and attract all solution orbits at an exponential rate, their corresponding inertial manifold forms are powerful tools which could study the property of finite dynamical system about the dissipative evolution equation. Under the restriction of inertial manifold, a infinite dimension dynamical system could be transformed to finite dimension, therefore, the inertial manifolds become an important bridge which 
can contact finite dimensional dynamical system and infinite dimensional dynamical system, many scholars have done a great deal of research, we could refer to $([2]-[8])$.

Guigui Xu, Libo Wang and Guoguang Lin [9] studied global attractor and inertial manifold for the strongly damped wave equations

$$
\left\{\begin{array}{l}
u_{t t}-\alpha \Delta u+\beta \Delta^{2} u-\gamma \Delta u_{t}+g(u)=f(x, t),(x, t) \in \Omega \times R^{+}, \\
u(x, 0)=u_{0}(x), u_{t}(x, 0)=u_{1}(x), x \in \Omega, \\
\left.u\right|_{\partial \Omega}=0,\left.\Delta u\right|_{\partial \Omega}=0,(x, t) \in \partial \Omega \times R^{+} .
\end{array}\right.
$$

The assumption of $g(u)$ satisfies the following conditions:

$\left(\mathrm{H}_{1}\right) \lim _{|s| \rightarrow \infty} \inf \frac{G(s)}{s^{2}} \geq 0, s \in R, G(s)=\int_{0}^{s} g(r) \mathrm{d} r$;

$\left(\mathrm{H}_{2}\right)$ There is a positive constant $C_{1}$, such that $\lim _{|s| \rightarrow \infty} \inf \frac{s g(s)-C_{1} G(s)}{s^{2}} \geq 0, s \in R$.

Under these reasonable assumptions, according to Hadamard's graph transformation method, the existence of the inertial manifolds for the equation is obtained.

Zhijian Yang and Zhiming Liu [10] studied the existence of exponential attractor for the Kirchhoff equations with strong nonlinear strongly dissipation and supercritical nonlinearity

$$
u_{t t}-\sigma\left(\|\nabla u\|^{2}\right) \Delta u_{t}-\phi\left(\|\nabla u\|^{2}\right) \Delta u+f(u)=h(x) .
$$

The main result was that the nonlinearity $f(u)$ is of supercritical growth and they established an exponential attractor in natural energy space by using a new method based on the weak quasi-stability estimates.

Ruijin Lou, Penghui Lv, Guoguang Lin [11] studied the exponential attractor and inertial manifold of a higher-order kirchhoff equations

$$
\begin{gathered}
u_{t t}+M\left(\left\|\nabla^{m} u\right\|_{p}^{p}\right)(-\Delta)^{2 m} u+\beta(-\Delta)^{2 m} u_{t}+g(u)=f(x), \\
u(x, t)=0, \frac{\partial^{i} u}{\partial v^{i}}=0, i=1,2, \cdots, 2 m-1, x \in \partial \Omega, \\
u(x, 0)=u_{0}(x), u_{t}(x, 0)=u_{1}(x), x \in \Omega, t>0 .
\end{gathered}
$$

where $\Omega$ is finite region of $R^{n}, \partial \Omega$ is smooth boundary, $u_{0}(x)$ and $u_{1}(x)$ is initial value, $(-\Delta)^{m} u_{t}$ is strongly damped term, $\phi$ is stress term, $g(u)$ is nonlinear source term.

On the basis of reference [11], the stress term $\left\|D^{m} u\right\|^{2}$ is extended to $\left\|D^{m} u\right\|_{p}^{p}$, this paper studied the long-time dynamic behavior of a class of generalized Kirchhoff equation. Firstly, the existence of the exponential attractor of this equation is proved. Furthermore, the existence of a family of inertial manifold is proved by using Hadamard's graph transformation method, more relevant research can be referred to ([12]-[17]). 
In this paper, we study the existence of exponential attractors and a family of the inertial manifolds for a class of generalized Kirchhoff-type equation with damping term:

$$
\begin{gathered}
u_{t t}+M\left(\left\|\nabla^{m} u\right\|_{p}^{p}\right)(-\Delta)^{2 m} u+\beta(-\Delta)^{2 m} u_{t}+g(u)=f(x), \\
u(x, t)=0, \frac{\partial^{i} u}{\partial v^{i}}=0, i=1,2, \cdots, 2 m-1, x \in \partial \Omega \\
u(x, 0)=u_{0}(x), u_{t}(x, 0)=u_{1}(x), x \in \Omega, t>0 .
\end{gathered}
$$

where $m>1, \quad p \geq 2, \Omega \subset R^{n}(n \geq 1)$ is a bounded domain with a smooth boundary $\partial \Omega, M(s) \in C^{2}\left([0,+\infty) ; R^{+}\right)$is a real function, $\beta(-\Delta)^{2 m} u_{t}(\beta>0)$ denotes strong damping term, $g(u)$ is nonlinear source term, $f(x)$ denotes the external force term. The assumption of $M(s)$ and $g(u)$ as follow:

(A1) $g(u) \in C^{\infty}(R)$

(A2) $M(s) \in C^{2}\left([0,+\infty), R^{+}\right), 1 \leq \mu_{0}<M(s)<\mu_{1}, \mu=\left\{\begin{array}{l}\mu_{0}, \frac{\mathrm{d}}{\mathrm{d} t}\left\|\nabla^{2 m} u\right\|^{2} \geq 0, \\ \mu_{1}, \frac{\mathrm{d}}{\mathrm{d} t}\left\|\nabla^{2 m} u\right\|^{2}<0 .\end{array}\right.$

where $\mu, \mu_{0}, \mu_{1}$ are constant, $\lambda_{1}$ is the first eigenvalue of $-\Delta$ with homogeneous Dirichlet boundary conditions on $\Omega$.

For convenience, define the following spaces and notations $H=L^{2}(\Omega)$, $H_{0}^{2 m}(\Omega)=H^{2 m}(\Omega) \cap H_{0}^{1}(\Omega), H_{0}^{4 m}(\Omega)=H^{4 m}(\Omega) \cap H_{0}^{1}(\Omega)$, $H_{0}^{2 m+k}(\Omega)=H^{2 m+k}(\Omega) \cap H_{0}^{1}(\Omega), \quad E_{0}=H^{2 m}(\Omega) \times L^{2}(\Omega)$, $E_{k}=H_{0}^{2 m+k}(\Omega) \times H_{0}^{k}(\Omega), \quad(k=1,2, \cdots, 2 m), \quad f(x) \in L^{2}(\Omega) .(\cdot, \cdot)$ and $\|\cdot\|$ represent the inner product and norms of $H$ respectively, i.e.

$(u, v)=\int_{\Omega} u(x) v(x) \mathrm{d} x, \quad(u, u)=\|u\|^{2},\|\cdot\|=\|\cdot\|_{L^{2}(\Omega)},\|\cdot\|_{P}=\|\cdot\|_{L^{P}(\Omega)}$, $\|\cdot\|_{\infty}=\|\cdot\|_{L^{\infty}(\Omega)} \cdot$

\section{Exponential Attractors}

For brevity, define the inner product and norms as follow: $\forall U_{i}=\left(u_{i}, v_{i}\right) \in E_{0}, i=1,2$,

$$
\begin{gathered}
\left(U_{1}, U_{2}\right)=\left(\nabla^{2 m} u_{1}, \nabla^{2 m} u_{2}\right)+\left(v_{1}, v_{2}\right), \\
\|U\|_{E_{0}}^{2}=(U, U)_{E_{0}}=\left\|\nabla^{2 m} u\right\|^{2}+\|v\|^{2} .
\end{gathered}
$$

Let $U=(u, v) \in E_{0}, \quad v=u_{t}+\varepsilon u, \quad 0<\varepsilon \leq \min \left\{4-\beta, 1-\frac{\alpha}{\beta \lambda_{1}^{2 m}}, \frac{\alpha}{1+\lambda_{1}^{-2 m}}\right\}$, we can get the Equation (1.1) is equivalent to the following evolution equation

$$
U_{t}+G(U)=F(U)
$$

where

$$
G=\left(\begin{array}{cc}
\varepsilon & -I \\
\varepsilon^{2}-\beta \varepsilon(-\Delta)^{2 m}+M\left(\left\|\nabla^{m} u\right\|_{p}^{p}\right)(-\Delta)^{2 m} & \beta(-\Delta)^{2 m}-\varepsilon
\end{array}\right)
$$




$$
F(U)=\left(\begin{array}{c}
0 \\
f(x)-g(u)
\end{array}\right)
$$

Then, we will use the following notations. Let $E_{0}, E_{k}$ are two Hilbert spaces, we have $E_{k} \hookrightarrow E_{0}$ with dense and continuous injection, and $E_{k} \hookrightarrow E_{0}$ is compact. Let $S(t)$ is a map from $E_{0}\left(E_{k}\right)$ into $E_{0}\left(E_{k}\right)$.

In the following definitions, $k=1,2, \cdots, 2 m$.

Definition 2.1. [14] The semigroup $S(t)$ possesses a $\left(E_{k}, E_{0}\right)$-compact attractor $A_{k}$, If it exists a compact set $A_{k} \subset E_{0}, A_{k}$ attracts all bounded subsets of $E_{k}$, and under the function of $S(t), A_{k}$ is an invariant set, i.e. $S(t) A_{k}=A_{k}, \forall t \geq 0$.

Definition 2.2. [14] If $A_{k} \subseteq M_{k} \subseteq B_{k}$ and 1) $\left.S(t) M_{k} \subseteq M_{k}, \forall t \geq 0 ; 2\right)$ $M_{k}$ has finite fractal dimension, $\left.d_{F}\left(M_{k}\right)<+\infty ; 3\right)$ there exist universal constants $c_{1}>0, c_{2}>0$, such that $\operatorname{dist}\left(S(t) B_{k}, M_{k}\right) \leq c_{1} \mathrm{e}^{-c_{2} t}, t>0$, where $\operatorname{dist}_{E_{0}}\left(A_{k}, B_{k}\right)=\sup _{x \in A_{k}} \inf _{y \in B_{k}}|x-y|_{E_{0}}, B_{k} \subset E_{k}$ is the positive invariant set of $S(t)$, the compact set $M_{k} \subset E_{0}$ is called a $\left(E_{k}, E_{0}\right)$-exponential attractor for the system $\left(S(t), B_{k}\right)$.

Definition 2.3. [14] if there exists limited function $l(t)$, such that

$$
\|S(t) u-S(t) v\|_{E_{0}} \leq l(t)\|u-v\|_{E_{0}}, \forall(u, v) \in B_{k} .
$$

Then the semigroup $S(t)$ is Lipschitz continuous in $B_{k}$.

Definition 2.4. [14] If $\delta \in\left(0, \frac{1}{8}\right)$ and exists an orthogonal projection $P_{N}=P_{N}(\delta)$ of rank $N=N(\delta)$ such that for every $(u, v) \in B_{k}$,

$$
\left\|S\left(t_{*}\right) u-S\left(t_{*}\right) v\right\|_{E_{0}} \leq \delta\|u-v\|_{E_{0}},
$$

or

$$
\left\|Q_{N}\left(S\left(t_{*}\right) u-S\left(t_{*}\right) v\right)\right\|_{E_{0}} \leq\left\|P_{N}\left(S\left(t_{*}\right) u-S\left(t_{*}\right) v\right)\right\|_{E_{0}}
$$

Then $S(t)$ is said to satisfy the discrete squeezing property, where $Q_{N}=I-P_{N}$.

Theorem 2.1. [15] Assume that 1) $S(t)$ possesses a $\left(E_{k}, E_{0}\right)$-compact attractor $\left.A_{k} ; 2\right)$ it exists a positive invariant compact set $B_{k} \subset E_{0}$ of $\left.S(t) ; 3\right)$ $S(t)$ is a Lipschitz continuous map with Lipschitz constant $l$ on $B_{k}$, and satisfies the discrete squeezing property on $B_{k}$. Then $S(t)$ has a $\left(E_{k}, E_{0}\right)$-exponential attractor $M_{k}$, and $M_{k} \supseteq A_{k}$ on $B_{k}$, and $M_{k}=\bigcup_{0 \leq t \leq t_{*}} S(t) M_{*}$, $M_{*}=A_{k} \cup\left(\bigcup_{j=1}^{\infty} \bigcup_{i=1}^{\infty} S\left(t_{*}\right)^{j}\left(E^{(i)}\right)\right)$. Moreover, the fractal dimension of $M_{k}$ satisfies $d_{F}\left(M_{k}\right) \leq c_{3} N_{0}+1, \operatorname{dist}_{E_{0}}\left(S(t) B, M_{k}\right) \leq c_{1} \mathrm{e}^{-c_{2} t}$, where $N_{0}$ is the smallest $N$ which make the discrete squeezing property established.

Proposition 2.1. [15] There is $t_{0}\left(D_{k}\right)$ such that $B_{k}=\overline{\bigcup_{0 \leq t \leq t_{0}\left(D_{k}\right)} S(t) D_{k}}$ is 
the positive invariant set of $S(t)$ in $E_{0}$, and $B_{k}$ attracts all bounded subsets of $E_{k}$, where $B_{k}$ is a closed bounded absorbing set for $S(t)$ in $E_{k}$.

Theorem 2.2. [16] Assuming the stress term $M(s)$ and the nonlinear term $g(u)$ satisfies the condition (A1)-(A2), $f \in H,\left(u_{0}, v_{0}\right) \in E_{k}$, then problem (1.1)-(1.3) admits a unique solution $(u, v) \in L^{\infty}\left(R^{+} ; E_{k}\right)$. This solution possesses the following properties:

$$
\|(u, v)\|_{E_{0}}^{2}=\left\|\nabla^{2 m} u\right\|^{2}+\|v\|^{2} \leq c\left(r_{0}\right), \quad\|(u, v)\|_{E_{k}}^{2}=\left\|\nabla^{2 m+k} u\right\|^{2}+\left\|\nabla^{2 m} v\right\|^{2} \leq c\left(r_{1}\right) .
$$

We denote the solution in Theorem 2.1 by $S(t)\left(u_{0}, v_{0}\right)=(u(t), v(t))$. Then $S(t)$ composes a continuous semigroup in $E_{0}$. According to Theorem 2.1, we have the ball

$$
\begin{gathered}
D_{0}=\left\{(u, v) \in E_{0}:\|(u, v)\|_{E_{0}}^{2}=\left\|\nabla^{2 m} u\right\|^{2}+\|v\|^{2} \leq c\left(r_{0}\right)\right\}, \\
D_{k}=\left\{(u, v) \in E_{k}:\|(u, v)\|_{E_{k}}^{2}=\left\|\nabla^{2 m+k} u\right\|^{2}+\left\|\nabla^{2 m} v\right\|^{2} \leq c\left(r_{1}\right)\right\} .
\end{gathered}
$$

are absorbing sets of $S(t)$ in $E_{0}$ and $E_{k}$ respectively. From Proposition 2.1

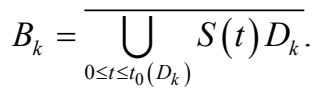

is a positive invariant compact set of $S(t)$ in $E_{0}$, and absorbs all of the bounded subsets $D_{k}$ in $E_{k}$. According to reference [15] and theorem 2.1, we can get the semigroup $\{S(t)\}_{t \geq 0}$ possesses $\left(E_{k}, E_{0}\right)$-compact global attractor

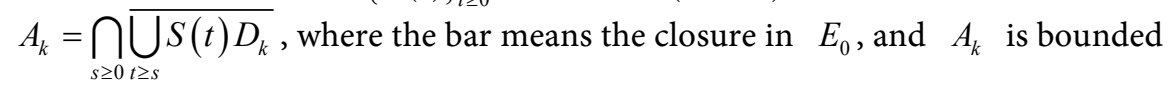
in $E_{k}$.

Lemma 2.1. For any $U=(u, v) \in E_{0}$,

$$
(G(U), U)_{E_{0}} \geq a_{1}\|U\|_{E_{0}}^{2}+a_{2}\left\|\nabla^{2 m} v\right\|^{2} .
$$

Proof. By (2.1) and (2.2), we have

$$
\begin{aligned}
(G(U), U)_{E_{0}}= & \varepsilon\left\|\nabla^{2 m} u\right\|^{2}-\left(\nabla^{2 m} v, \nabla^{2 m} u\right)+(\alpha-\varepsilon)\|v\|^{2}+\varepsilon^{2}(u, v)-\alpha\|v\|^{2} \\
& -\beta \varepsilon\left(\nabla^{2 m} u, \nabla^{2 m} v\right)+\beta\left\|\nabla^{m} v\right\|^{2}+M\left(\left\|\nabla^{m} u\right\|_{p}^{p}\right)\left(\nabla^{2 m} u, \nabla^{2 m} v\right)
\end{aligned}
$$

By using Holder's inequality, Young's inequality and Poincare's inequality and the condition (A2), we have,

$$
\begin{gathered}
\varepsilon^{2}(u, v)=-\varepsilon^{2} \lambda_{1}^{-m}\left\|\nabla^{2 m} u\right\|\|v\| \geq-\frac{\varepsilon^{2}}{4}\left\|\nabla^{2 m} u\right\|^{2}-\varepsilon^{2} \lambda_{1}^{-2 m}\|v\|^{2} \\
M\left(\left\|\nabla^{m} u\right\|_{p}^{p}-\beta \varepsilon-1\right)\left(\nabla^{2 m} u, \nabla^{2 m} v\right) \geq\left(\mu_{0}-\beta \varepsilon-1\right)\left(\frac{\left\|\nabla^{2 m} u\right\|^{2}}{4}+\left\|\nabla^{2 m} v\right\|^{2}\right) \\
=-\beta \varepsilon\left(\frac{\left\|\nabla^{2 m} u\right\|^{2}}{4}+\left\|\nabla^{2 m} v\right\|^{2}\right)
\end{gathered}
$$

Substitute inequality (2.12)-(2.13) into Equation (2.11), we get 


$$
\begin{aligned}
(G(U), U)_{E_{0}} \geq & \left(\varepsilon-\frac{\beta \varepsilon}{4}-\frac{\varepsilon^{2}}{4}\right)\left\|\nabla^{2 m} u\right\|^{2}+\left(\alpha-\varepsilon-\varepsilon^{2} \lambda_{1}^{-2 m}\right)\|v\|^{2} \\
& +\left(\beta-\beta \varepsilon-\alpha \lambda_{1}^{-2 m}\right)\left\|\nabla^{2 m} v\right\|^{2}
\end{aligned}
$$

According to the assumption, we can get $\varepsilon-\frac{\beta \varepsilon}{4}-\frac{\varepsilon^{2}}{4}>0$,

$$
\begin{gathered}
\alpha-\varepsilon-\varepsilon^{2} \lambda_{1}^{-2 m}>0, \quad \beta-\beta \varepsilon-\alpha \lambda_{1}^{-2 m}>0 . \text { Let } \\
a_{1}=\min \left\{\varepsilon-\frac{\beta \varepsilon}{4}-\frac{\varepsilon^{2}}{4}, \alpha-\varepsilon-\varepsilon^{2} \lambda_{1}^{-2 m}\right\}, a_{2}=\beta-\beta \varepsilon-\alpha \lambda_{1}^{-2 m} \text {, so we can get } \\
(G(U), U)_{E_{0}} \geq a_{1}\|U\|_{E_{0}}^{2}+a_{2}\left\|\nabla^{2 m} v\right\|^{2} .
\end{gathered}
$$

The Lemma 2.1 is proved. Then we prove the Lipschitz property and the discrete squeezing property of $S(t)$.

Set $S(t) U_{0}=U(t)=(u(t), v(t))^{\mathrm{T}}$, where $v=u_{t}(t)+\varepsilon u(t)$; and $S(t) V_{0}=V(t)=(\hat{u}(t), \hat{v}(t))^{\mathrm{T}}$, where $\hat{v}(t)=\hat{u}_{t}(t)+\varepsilon \hat{u}(t) ;$ let $Y(t)=S(t) U_{0}-S(t) V_{0}=U(t)-V(t)=(w(t), z(t))^{\mathrm{T}}$, where $z(t)=w_{t}(t)+\varepsilon w(t), \quad w(t)=u(t)-\hat{u}(t), \quad w_{t}(t)=v(t)-\hat{v}(t)$, then $Y(t)$ satisfies

$$
\begin{gathered}
Y_{t}+G(U)-G(V)-(0, g(u)-g(\hat{u}))^{\mathrm{T}}=0, \\
Y(0)=U_{0}-V_{0} .
\end{gathered}
$$

Lemma 2.2. (Lipschitz property). For $\forall U_{0}, V_{0} \in B_{k}$ and $t \geq 0$,

$$
\left\|S(t) U_{0}-S(t) V_{0}\right\|_{E_{0}} \leq \mathrm{e}^{\gamma t}\left\|U_{0}-V_{0}\right\|_{E_{0}}^{2} .
$$

Proof. Taking the inner product of the Equation (2.16) with $Y(t)$ in $E_{0}$, we can get

$$
\frac{1}{2} \frac{\mathrm{d}}{\mathrm{d} t}\|Y(t)\|^{2}+(G(U)-G(V), Y(t))+(g(u)-g(\hat{u}), z(t))=0 .
$$

Similar to Lemma 2.1, we have

$$
(G(U)-G(V), Y(t))_{E_{0}} \geq a_{1}\|Y(t)\|_{E_{0}}^{2}+a_{2}\left\|\nabla^{2 m} z(t)\right\|_{E_{0}}^{2} .
$$

By using the condition (A1) Young's inequality Poincare's inequality and differential mean value theorem, we get

$$
\begin{aligned}
|(g(u)-g(\hat{u}), z(t))| & \leq\left|g^{\prime}(\xi)\right|\|w(t)\|\|z(t)\| \leq c_{4} \lambda_{1}^{-m}\left\|\nabla^{2 m} w(t)\right\|\|z(t)\| \\
& \leq \frac{c_{4} \lambda_{1}^{-m}}{2}\left(\left\|\nabla^{2 m} w(t)\right\|^{2}+\|z(t)\|^{2}\right)=\frac{c_{4} \lambda_{1}^{-m}}{2}\|Y(t)\|^{2} .
\end{aligned}
$$

Where $\xi=\theta+(1-\theta) \hat{u}, 0<\theta<1$.

Substitute inequality (2.20)-(2.21) into equation (2.19), we get

$$
\frac{\mathrm{d}}{\mathrm{d} t}\|Y(t)\|^{2}+2 a_{1}\|Y(t)\|_{E_{0}}^{2}+2 a_{2}\left\|\nabla^{2 m} z(t)\right\|_{E_{0}}^{2} \leq c_{4} \lambda_{1}^{-m}\|Y(t)\|^{2} .
$$

We can get 


$$
\frac{\mathrm{d}}{\mathrm{d} t}\|Y(t)\|^{2} \leq c_{4} \lambda_{1}^{-m}\|Y(t)\|^{2} .
$$

According to Gronwall's inequality, we have

$$
\|Y(t)\|^{2} \leq \mathrm{e}^{c_{4} \lambda_{1}^{-m} t}\|Y(0)\|^{2}=\mathrm{e}^{\gamma t}\|Y(0)\|^{2} .
$$

where $\gamma=c_{4} \lambda_{1}^{-m}$. Therefore, we get

$$
\left\|S(T) U_{0}-S(T) V_{0}\right\|_{E_{0}} \leq \mathrm{e}^{\gamma t}\left\|U_{0}-V_{0}\right\|_{E_{0}}^{2} .
$$

The Lemma 2.2 is proved.

Now, we define the operator $-\Delta: D(-\Delta) \rightarrow H^{4 m}$, the domain of definition is $D(-\Delta)=H^{2}(\Omega) \cap H_{0}^{1}(\Omega)$, obviously, $-\Delta$ is an unbounded self-adjoint closed positive operator, and $(-\Delta)^{-1}$ is compact, we find by elementary spectral theory the existence of an orthonormal basis of $\mathrm{H}$ consisting of eigenvectors $w_{j}$ of $-\Delta$, such that:

$$
\left\{\begin{array}{l}
(-\Delta) w_{j}=\lambda_{j} w_{j}, \quad j=1,2, \cdots, \\
0<\lambda_{1} \leq \lambda_{2} \leq \cdots \quad \lambda_{j} \rightarrow \infty \text { as } j \rightarrow \infty .
\end{array}\right.
$$

For a given integer $n, 0<n \leq N$ we denote by $P_{n}$ the orthogonal projection of $H^{4 m}$ onto the space spanned by $w_{1}, \cdots, w_{n}$ i.e.

$p=p_{n}=H^{4 m} \rightarrow \operatorname{span}\left\{w_{1}, w_{2}, \cdots, w_{n}\right\}$, let $Q_{n}=I-P_{n}$. Then we have

$$
\begin{gathered}
\left\|(-\Delta)^{2 m} u\right\| \geq \lambda_{n+1}^{2 m}\|u\|, \quad \forall u \in Q=Q_{n}\left(H^{4 m}(\Omega) \cap H_{0}^{1}(\Omega)\right), \\
\left\|Q_{n} u\right\| \leq\|u\|, \quad u \in H .
\end{gathered}
$$

where $\|u\|^{2} \leq \lambda_{n+1}^{-4 m}\|u\|_{D\left((-\Delta)^{2 m}\right)}^{2}$.

Lemma 2.3. For any $U_{0}, V_{0} \in B_{k}, \forall n_{0} \in N^{*}, n_{0} \leq N$, Let

$$
Q_{n_{0}}(t)=Q_{n_{0}}(U(t)-V(t))=Q_{m_{0}} Y(t)=\left(\omega_{n_{0}}, z_{n_{0}}\right)^{\mathrm{T}},
$$

then we have

$$
\left\|Y_{n_{0}}(t)\right\|_{E_{0}}^{2} \leq\left(\mathrm{e}^{-2 a_{1} t}+\frac{c_{2} \lambda_{n_{0}+1}^{-m}}{2 a_{1}+\gamma} \mathrm{e}^{\gamma t}\right)\|Y(0)\|_{E_{0}}^{2},
$$

Proof. Taking projection operator $Q_{n_{0}}$ in (2.16), we have

$$
Y_{n_{0} t}(t)+Q_{n_{0}}(G(U)-G(V))+\left(0, Q_{n_{0}}(g(u)-g(\hat{u}))\right)^{\mathrm{T}}=0 .
$$

Taking the inner product $(\cdot, \cdot)_{E_{0}}$ in (2.31) with $Y_{n_{0}}(t)$, we get

$$
\frac{1}{2} \frac{\mathrm{d}}{\mathrm{d} t}\left\|Y_{n_{0}}(t)\right\|^{2}+a_{1}\left\|Y_{n_{0}}(t)\right\|^{2}+a_{2}\left\|\nabla^{2 m} z_{n_{0}}(t)\right\|^{2}+Q_{n_{0}}\left(g(u)-g(\hat{u}), z_{n_{0}}(t)\right)=0 .
$$

According to (A1) and Young inequality, we have

$$
\begin{aligned}
& \left|Q_{n_{0}}\left(g(u)-g(\hat{u}), z_{n_{0}}(t)\right)\right| \\
& \leq\left|g^{\prime}\left(\xi^{\prime}\right)\right|\left\|w_{n_{0}}(t)\right\|\left\|z_{n_{0}}(t)\right\| \leq c_{5} \lambda_{n_{0}+1}^{-m}\left\|\nabla^{2 m} w_{n_{0}}(t)\right\|\left\|z_{n_{0}}(t)\right\| \\
& \leq \frac{c_{5} \lambda_{n_{0}+1}^{-m}}{2}\left(\left\|\nabla^{2 m} w_{n_{0}}(t)\right\|^{2}+\left\|z_{n_{0}}(t)\right\|^{2}\right)=\frac{c_{5} \lambda_{n_{0}+1}^{-m}}{2}\left\|Y_{n_{0}}(t)\right\|^{2} .
\end{aligned}
$$


where $\xi^{\prime}=\theta_{n_{0}}+\left(1-\theta_{n_{0}}\right) \hat{u}, 0<\theta_{n_{0}}<1$.

Together with (2.32)-(2.33) and Lemma 2.2, it follows

$$
\begin{aligned}
\frac{\mathrm{d}}{\mathrm{d} t}\left\|Y_{n_{0}}\right\|_{E_{0}}^{2}+2 a_{1}\left\|Y_{n_{0}}(t)\right\|^{2} & \leq c_{5} \lambda_{n_{0}+1}^{-m}\left\|Y_{n_{0}}(t)\right\|^{2}=c_{5} \lambda_{n_{0}+1}^{-m}\left\|S(t) U_{0}-S(t) V_{0}\right\|^{2} \\
& \leq c_{5} \lambda_{n_{0}+1}^{-m} \mathrm{e}^{\gamma t}\left\|U_{0}-V_{0}\right\|^{2}=c_{5} \lambda_{n_{0}+1}^{-m} \mathrm{e}^{\gamma t}\|Y(0)\|^{2} .
\end{aligned}
$$

By using Gronwall's inequality, we get

$$
\left\|Y_{n_{0}}(t)\right\|^{2} \leq\|Y(0)\|^{2} \mathrm{e}^{-2 a_{1} t}+\frac{c_{5} \lambda_{n_{0}+1}^{-m}}{2 a_{1}+\gamma} \mathrm{e}^{\gamma t}\|Y(0)\|^{2}=\left(\mathrm{e}^{-2 a_{1} t}+\frac{c_{5} \lambda_{n_{0}+1}^{-m}}{2 a_{1}+\gamma} \mathrm{e}^{\gamma t}\right)\|Y(0)\|^{2} .
$$

The Lemma 2.3 is proved.

Lemma 2.4. (Discrete squeezing property). For any $U_{0}, V_{0} \in B_{k}, \tau^{*} \geq 0$, if

$$
\left\|P_{n_{0}}\left(S\left(\tau^{*}\right) U_{0}-S\left(\tau^{*}\right) V_{0}\right)\right\|_{E_{0}} \leq\left\|\left(I-P_{n_{0}}\right)\left(S\left(\tau^{*}\right) U_{0}-S\left(\tau^{*}\right) V_{0}\right)\right\|_{E_{0}},
$$

then

$$
\left\|S\left(\tau^{*}\right) U_{0}-S\left(\tau^{*}\right) V_{0}\right\|_{E_{0}} \leq \frac{1}{8}\left\|U_{0}-V_{0}\right\|_{E_{0}} .
$$

Proof. If $\left\|P_{n_{0}}\left(S\left(\tau^{*}\right) U_{0}-S\left(\tau^{*}\right) V_{0}\right)\right\|_{E_{0}} \leq\left\|\left(I-P_{n_{0}}\right)\left(S\left(\tau^{*}\right) U_{0}-S\left(\tau^{*}\right) V_{0}\right)\right\|_{E_{0}}$, then

$$
\begin{aligned}
& \left\|S\left(\tau^{*}\right) U_{0}-S\left(\tau^{*}\right) V_{0}\right\|_{E_{0}}^{2} \\
& \leq\left\|\left(I-P_{n_{0}}\right)\left(S\left(\tau^{*}\right) U_{0}-S\left(\tau^{*}\right) V_{0}\right)\right\|_{E_{0}}^{2}+\left\|P_{n_{0}}\left(S\left(\tau^{*}\right) U_{0}-S\left(\tau^{*}\right) V_{0}\right)\right\|_{E_{0}}^{2} \\
& \leq 2\left\|\left(I-P_{n_{0}}\right)\left(S\left(\tau^{*}\right) U_{0}-S\left(\tau^{*}\right) V_{0}\right)\right\|_{E_{0}}^{2} \leq 2\left(\mathrm{e}^{-2 a_{1} \tau^{*}}+\frac{c_{5} \lambda_{n_{0}+1}^{-m}}{2 a_{1}+\gamma} \mathrm{e}^{\gamma \tau^{*}}\right)\left\|U_{0}-V_{0}\right\|_{E_{0}}^{2} .
\end{aligned}
$$

Let $\tau^{*}$ be large enough,

$$
\mathrm{e}^{-2 a_{1} \tau^{*}} \leq \frac{1}{256}
$$

Also let $n_{0}$ be large enough, we get

$$
\frac{c_{5} \lambda_{n_{0}+1}^{-m}}{2 a_{1}+\gamma} \mathrm{e}^{\gamma \tau^{*}} \leq \frac{1}{256} .
$$

Substitute inequality (2.39)-(2.40) into Equation (2.38), we get

$$
\left\|S\left(\tau^{*}\right) U_{0}-S\left(\tau^{*}\right) V_{0}\right\|_{E_{0}}^{2} \leq \frac{1}{8}\left\|U_{0}-V_{0}\right\|_{E_{0}}^{2} .
$$

The Lemma 2.4 is proved.

Theorem 2.3. Let (A1), (A2) be in force, assume that $f \in H$, $\left(u_{0}, v_{0}\right) \in E_{0}\left(E_{k}\right),(k=1,2, \cdots, 2 m)$, then the semigroup $S(t)$ determined by (1.1)-(1.3) possesses an $\left(E_{k}, E_{0}\right)$-exponential attractor $M_{k}$ on $B$,

$$
M_{k}=\bigcup_{0 \leq t \leq \tau^{*}} S(t)\left(A_{k} \cup\left(\bigcup_{j=1}^{\infty} \bigcup_{i=1}^{\infty} S\left(\tau^{*}\right)^{j}\left(E^{(i)}\right)\right)\right),
$$

The fractal dimension of $M_{k}$ satisfies 


$$
d_{F}\left(M_{k}\right) \leq c_{3} N_{0}+1 .
$$

Proof. According to Theorem 2.1, Lemma 2.2 and Lemma 2.4, Theorem 2.2 is easily proven.

\section{Inertial Manifolds}

Next, we will prove the existence of inertial manifolds when $\mathrm{N}$ is large enough by using graph norm transformation method.

Definition 3.1. [17] Assume $S=S(t)_{t \geq 0}$ is a solution semigroup of Banach space $E_{k}=H_{0}^{2 m+k}(\Omega) \times H_{0}^{k}(\Omega)(k=1,2, \cdots, 2 m)$, then a family of inertial manifolds $\mu_{k}$ is a subset of $E_{k}$ and satisfies the following three properties:

1) $\mu_{k}$ is finite dimensional Lipschitz manifold of $E_{k}$;

2) $\mu_{k}$ is positively invariant for the semigroup $\{S(t)\}_{t \geq 0}$, i.e. $\forall u_{0} \in \mu_{k}$, $S(t) u_{0} \subset \mu_{k}, \quad \forall t \geq 0$;

3) $\mu_{k}$ attracts exponentially all the orbits of the solution, i.e. $\exists \vartheta>0$, for $\forall u \in E_{k}, \quad \exists k>0$, such that

$$
\operatorname{dist}(S(t) u, u) \leq k \cdot \mathrm{e}^{-\vartheta t}, t \geq 0 .
$$

Lemma 3.1. Let $\Lambda: E_{k} \rightarrow E_{k}$ be an operator and assume that $F \in C_{b}\left(E_{k}, E_{k}\right)$ satisfies the Lipschitz condition

$$
\|F(U)-F(U)\|_{E_{k}} \leq l_{F}\|U-V\|_{E_{k}}, \quad U, V \in E_{k} .
$$

The operator $\Lambda$ is said to satisfy the spectral gap condition relative to $F$, if the point spectrum of the operator $\Lambda$ can be divided into two parts $\sigma_{1}$ and $\sigma_{2}$, of which $\sigma_{1}$ is finite, and we have

$$
\Lambda_{1}=\sup \left\{\operatorname{Re} \lambda \mid \lambda \in \sigma_{1}\right\}, \quad \Lambda_{2}=\inf \left\{\operatorname{Re} \lambda \mid \lambda \in \sigma_{2}\right\},
$$

and $E_{k_{i}}=\operatorname{span}\left\{\omega_{j} \mid j \in \sigma_{i}, i=1,2\right\}$.

Then

$$
\Lambda_{2}-\Lambda_{1}>4 l_{F}
$$

and the orthogonal decomposition

$$
E_{k}=E_{k_{1}} \oplus E_{k_{2}},
$$

Then $P_{1}: E_{k} \rightarrow E_{k_{1}}$ and $P_{2}: E_{k} \rightarrow E_{k_{2}}$ are both continuous orthogonal projections. The Lemma 3.1 is proved.

Lemma 3.2. Let the eigenvalues $\mu_{j}^{ \pm}(j \geq 1)$ is non-decreasing, and for $m \in N^{*}$, there exists $N \geq m$, such that $\mu_{N+1}^{-}$and $\mu_{N}^{-}$are consecutive adjacent values.

Lemma 3.3. The function $g(u)$ satisfies $g: H_{0}^{k}(\Omega) \rightarrow L^{2}(\Omega)$ which is uniformly bounded and globally Lipschitz continuous, and $l$ is the Lipschitz coefficient.

Proof. For $\forall u_{1}, u_{2} \in H_{0}^{k}(\Omega)$, we have

$$
\left\|g\left(u_{1}\right)-g\left(u_{2}\right)\right\|=\left\|g^{\prime}(\eta)\left(u_{1}-u_{2}\right)\right\| \leq\left\|g^{\prime}(\eta)\right\|_{\infty}\left\|u_{1}-u_{2}\right\|_{H_{k}},
$$

where $\eta \in\left(u_{1}, u_{2}\right)$, From the hypothesis (A1) and the differential mean value 
theorem, we know

$$
\left\|g\left(u_{1}\right)-g\left(u_{2}\right)\right\| \leq C_{6}\left\|u_{1}-u_{2}\right\|_{H_{0}^{k}},
$$

Let $l=C_{6}, \quad l$ is the Lipschitz coefficient.

Then we prove the existence of a family of the inertial manifold of this equation, Equation (1.1) is equivalent to the following first-order evolution equation:

$$
U_{t}+\Lambda U=F(U)
$$

where

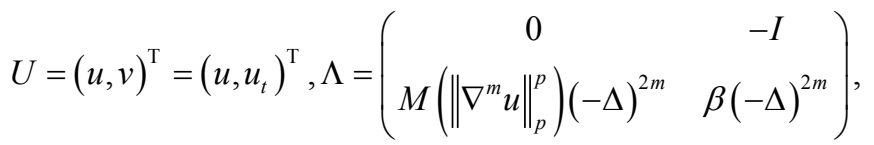

$$
\begin{aligned}
& F(U)=\left(\begin{array}{c}
0 \\
f(x)-g(u)
\end{array}\right) \\
& D(\Lambda)=\left\{u \in H^{4 m}(\Omega) \mid u \in H,(-\Delta)^{2 m} u \in H^{4 m}(\Omega)\right\} \times H^{2 m}(\Omega) \text {. }
\end{aligned}
$$

We consider in $E_{k}$ the usual graph norm, induced by the scalar product

$$
\langle U, V\rangle_{E_{k}}=\left(M \cdot \nabla^{2 m+k} u, \nabla^{2 m+k} \bar{y}\right)+(v, \bar{z}) \text {. }
$$

where $U=(u, v)^{\mathrm{T}}, V=(y, z)^{\mathrm{T}} \in E_{k}$, and $\bar{y}, \bar{z}$ respectively denote the conjugation of $y$ and $z$, and $v, z \in H_{0}^{2 m+k}(\Omega), u, y \in H_{0}^{2 m+k}(\Omega)$. Moreover, the operator $\Lambda$ is monotone, indeed, for $\forall U \in D(\Lambda)$, we have

$$
\begin{aligned}
& \langle\Lambda U, U\rangle_{E_{k}} \\
& =-\left(M \cdot \nabla^{2 m+k} u_{t}, \nabla^{2 m+k} \bar{u}\right)+\left(M\left(\left\|\nabla^{m} u\right\|_{p}^{p}\right)(-\Delta)^{2 m} u+\beta(-\Delta)^{2 m} u_{t}, \bar{v}\right) \\
& \geq-\left(M \cdot \nabla^{2 m+k} u_{t}, \nabla^{2 m+k} \bar{u}\right)+M\left(\nabla^{2 m+k} u, \nabla^{2 m+k} v\right)+\beta\left(-\Delta^{m} v,-\Delta^{m} \bar{v}\right) \\
& \geq \beta\left\|\nabla^{2 m} v\right\|^{2}>0 .
\end{aligned}
$$

so that $\Lambda$ is a Monotonically increasing operator and $\langle\Lambda U, U\rangle_{E_{k}}$ is real and nonnegative. To determine the eigenvalues of $\Lambda$, we observe that the eigenvalue equation

$$
\Lambda U=\lambda U, \quad U=(u, v)^{\mathrm{T}} \in E_{k}
$$

is equivalent to the system

$$
\left\{\begin{array}{l}
-v=\lambda u, \\
M\left(\left\|\nabla^{m} u\right\|_{p}^{p}\right)(-\Delta)^{2 m} u+\beta(-\Delta)^{2 m} v=0 .
\end{array}\right.
$$

Thus, we can get the eigenvalue problem

$$
\left\{\begin{array}{l}
\lambda^{2} u+M\left(\left\|\nabla^{m} u\right\|_{p}^{p}\right)(-\Delta)^{2 m} u-\beta \lambda(-\Delta)^{2 m} u=0, \\
\left.u\right|_{\partial \Omega}=\left.(-\Delta)^{2 m} u\right|_{\partial \Omega}=0 .
\end{array}\right.
$$

Using $(-\Delta)^{k} u$ with the first formula of (3.13) to take the inner product, and bring $u_{j}$ to the position of $u$, we can get 


$$
\lambda^{2}\left\|\nabla^{k} u\right\|^{2}+M\left(\left\|\nabla^{m} u\right\|_{p}^{p}\right)\left\|\nabla^{2 m+k} u\right\|^{2}-\beta \lambda\left\|\nabla^{2 m+k} u\right\|^{2}=0 .
$$

Regarding Equation (3.14) as a quadratic equation of one variable with respect to $\lambda$, for $\forall j \in N^{+}$and let $s=\left\|\nabla^{m} u\right\|_{p}^{p}, M=M(s)$, the corresponding eigenvalues of equation (3.11) are as follows:

$$
\lambda_{j}^{ \pm}=\frac{\beta \mu_{j} \pm \sqrt{\beta^{2} \mu_{j}^{2}-4 M \mu_{j}}}{2} .
$$

where $\mu_{j}(j \geq 1)$ is the eigenvalue of $(-\Delta)^{2 m}$ in $H_{0}^{2 m}(\Omega)$, and $\mu_{j}=\lambda_{1} j^{\frac{2 m}{n}}$. Because of $\beta$ is large enough, the eigenvalue of $\Lambda$ are all positive and real numbers, the corresponding eigenvalues have the form

$$
U_{j}^{ \pm}=\left(u_{j},-\lambda_{j}^{ \pm} u_{j}\right)
$$

For formula (3.15), for the convenience of later use, define the following formula

$$
\left\|\nabla^{2 m+k} u_{j}\right\|=\sqrt{\mu_{j}},\left\|\nabla^{k} u_{j}\right\|=1,\left\|\nabla^{-2 m-k} u_{j}\right\|=\frac{1}{\sqrt{\mu_{j}}}, k=1,2, \cdots, 2 m .
$$

Next, it will be proved that the eigenvalue of the operator $\Lambda$ satisfies the spectral interval condition.

Theorem 3.1 let $l$ is the Lipschitz constant of $g(u)$, assume $\mu_{j} \geq \frac{4 M(s)}{\beta^{2}}$, if $N_{1} \in Z^{+}$is large enough, when $N \geq N_{1}$, the following inequality holds

$$
\left(\mu_{N+1}+\mu_{N}\right)\left(\beta-\sqrt{\beta^{2} \mu_{j}^{2}-4 M(s)}\right) \geq \frac{8 l}{\sqrt{\beta^{2} \mu_{j}^{2}-4 M(s)}}+1 .
$$

Then, the operator $\Lambda$ satisfies the spectral gap condition of Lemma 3.1.

Proof. Because of all the eigenvalues of the operator $\Lambda$ are positive real numbers, $\beta \geq 2 \sqrt{\frac{M}{\mu_{j}}}$ and the sequence $\left\{\lambda_{j}^{-}\right\}_{j \geq 1}$ and $\left\{\lambda_{j}^{+}\right\}_{j \geq 1}$ are monotonically increasing. The theorem is proved in four steps below.

step 1 Since $\lambda_{j}^{ \pm}$is a non-decreasing sequence, according to Lemma 3.2, given $\mathrm{N}$, so that $\lambda_{N}^{-}$and $\lambda_{N+1}^{-}$are consecutive adjacent eigenvalues, the eigenvalues of the operator $\Lambda$ are decomposed into $\sigma_{1}$ and $\sigma_{2}$, where $\sigma_{1}$ is the finite parts, which are expressed as follows

$$
\begin{gathered}
\sigma_{1}=\left\{\lambda_{h}^{-}, \lambda_{j}^{+} \mid \max \left\{\lambda_{h}^{-}, \lambda_{j}^{+}\right\} \leq \lambda_{N}^{-}\right\}, \\
\sigma_{2}=\left\{\lambda_{h}^{+}, \lambda_{j}^{ \pm} \mid \lambda_{h}^{-} \leq \lambda_{N}^{-} \leq \min \left\{\lambda_{h}^{+}, \lambda_{j}^{ \pm}\right\}\right\} .
\end{gathered}
$$

step 2 The corresponding $E_{k}$ is decomposed into

$$
\begin{aligned}
& E_{k_{1}}=\operatorname{span}\left\{U_{h}^{-}, U_{j}^{ \pm} \mid \lambda_{h}^{-}, \lambda_{j}^{+} \in \sigma_{1}\right\}, \\
& E_{k_{2}}=\operatorname{span}\left\{U_{h}^{+}, U_{j}^{+} \mid \lambda_{h}^{-}, \lambda_{j}^{ \pm} \in \sigma_{2}\right\} .
\end{aligned}
$$

We aim at madding two orthogonal subspaces of $E_{k}$ and verifying the spec- 
tral gap condition (3.4) is true when $\Lambda_{1}=\lambda_{N}^{-}, \Lambda_{2}=\lambda_{N+1}^{-}$. Therefore, we further decompose $E_{k_{2}}=E_{S}+E_{R}$, i.e.

$$
\begin{gathered}
E_{S}=\operatorname{span}\left\{U_{h}^{-} \mid \lambda_{h}^{-} \leq \lambda_{N}^{-} \leq \lambda_{h}^{+}\right\}, \\
E_{R}=\operatorname{span}\left\{U_{R}^{+} \mid \lambda_{N}^{-} \leq \lambda_{j}^{ \pm}\right\} .
\end{gathered}
$$

And set $E_{N}=E_{k_{1}} \oplus E_{S}$. Note that $E_{k_{1}}$ and $E_{S}$ are finite dimensional, that $\lambda_{N}^{-} \in E_{k_{1}}, \lambda_{N+1}^{-} \in E_{R}$, and that the reason why $E_{k_{1}}$ is not orthogonal to $E_{k_{2}}$ is that, while it is orthogonal to $E_{R}, E_{k_{1}}$ is not orthogonal to $E_{S}$. We now introduce two functions $\Psi: E_{N} \rightarrow R$ and $\psi: E_{R} \rightarrow R$, defined by

$$
\begin{aligned}
\Phi(U, V)= & \beta\left(\nabla^{2 m+k} u, \nabla^{2 m+k} \bar{y}\right)+2 \beta\left(\nabla^{-2 m-k} \bar{z}, \nabla^{2 m} u\right) \\
& +2 \beta\left(\nabla^{-2 m-k} v, \nabla^{2 m} \bar{y}\right)+4\left(\nabla^{-2 m-k} v, \nabla^{-2 m-k} z\right) \\
& -4 M\left(\left\|\nabla^{m} u\right\|_{p}^{p}\right)\left(\nabla^{k} \bar{u}, \nabla^{k} y\right)+\left(2 \beta^{2}-\beta\right)\left(\nabla^{2 m+k} \bar{u}, \nabla^{2 m+k} y\right) . \\
\Psi(U, V)= & \left(\nabla^{2 m+k} u, \nabla^{2 m+k} \bar{y}\right)+\left(\nabla^{-2 m-k} \bar{z}, \nabla^{2 m+k} u\right)-\left(\nabla^{-2 m-k} v, \nabla^{2 m+k} \bar{y}\right) \\
& -4 M\left(\left\|\nabla^{m} u\right\|_{p}^{p}\right)\left(\nabla^{k} \bar{u}, \nabla^{k} y\right)+\left(\beta^{2}-1\right)\left(\nabla^{2 m+k} \bar{u}, \nabla^{2 m+k} y\right) .
\end{aligned}
$$

where $U=(u, v)^{\mathrm{T}}, V=(y, z)^{\mathrm{T}} \in E_{N}$, and $\bar{y}, \bar{z}$ are respectively the conjugates of $y, z$. We now show that $\Phi$ and $\Psi$ are positive definite. For $\forall U=(u, v) \in E_{N}$, we have

$$
\begin{aligned}
\Phi(U, V)= & \beta\left(\nabla^{2 m+k} u, \nabla^{2 m+k} \bar{u}\right)+2 \beta\left(\nabla^{-2 m-k} \bar{v}, \nabla^{2 m} u\right)+2 \beta\left(\nabla^{-2 m-k} v, \nabla^{2 m} \bar{u}\right) \\
& +4\left(\nabla^{-2 m-k} v, \nabla^{-2 m-k} v\right)-4 M\left(\left\|\nabla^{m} u\right\|_{p}^{p}\right)\left(\nabla^{k} \bar{u}, \nabla^{k} u\right) \\
& +\left(2 \beta^{2}-\beta\right)\left(\nabla^{2 m+k} \bar{u}, \nabla^{2 m+k} u\right) \\
\geq & \beta\left\|\nabla^{2 m+k} u\right\|^{2}-4\left\|\nabla^{-2 m-k} \bar{v}\right\|^{2}-\beta^{2}\left\|\nabla^{2 m+k} u\right\|^{2}-4 M(s)\left\|\nabla^{m+k} u\right\|^{2} \\
& +\left(2 \beta^{2}-\beta\right)\left\|\nabla^{2 m+k} u\right\|^{2}+4\left\|\nabla^{-2 m-k} \bar{v}\right\|^{2} \\
& =\beta^{2}\left\|\nabla^{2 m+k} u\right\|^{2}-4 \mu_{1}\left\|\nabla^{k} u\right\|^{2} \\
\geq & \left(\beta^{2} \mu_{j}^{2}-4 M(s)\right)\left\|\nabla^{k} u\right\|^{2} .
\end{aligned}
$$

When $\beta$ is large enough, we conclude that $\Phi(U, U) \geq 0$, i.e. $\Phi$ is positive definite. Similarly, for $\forall U=(u, v) \in E_{R}$, we have

$$
\begin{aligned}
\Psi(U, V)= & \left(\nabla^{2 m+k} u, \nabla^{2 m+k} \bar{u}\right)+\left(\nabla^{-2 m-k} \bar{v}, \nabla^{2 m+k} u\right)-\left(\nabla^{-2 m-k} v, \nabla^{2 m+k} \bar{u}\right) \\
& -4 M\left(\left\|\nabla^{m} u\right\|_{p}^{p}\right)\left(\nabla^{k} \bar{u}, \nabla^{k} u\right)+\left(\beta^{2}-1\right)\left(\nabla^{2 m+k} \bar{u}, \nabla^{2 m+k} u\right) \\
\geq & \left\|\nabla^{2 m+k} u\right\|^{2}-4 M(s)\left\|\nabla^{m+k} u\right\|^{2}+\left(\beta^{2}-1\right)\left\|\nabla^{2 m+k} u\right\|^{2} \\
\geq & \left(\beta^{2} \mu_{j}^{2}-4 M(s)\right)\left\|\nabla^{k} u\right\|^{2} .
\end{aligned}
$$

When $\beta$ is large enough, we conclude that $\Psi(U, U) \geq 0$, i.e. $\Psi$ is positive definite.

Thus $\Phi$ and $\Psi$ define a scalar product, respectively on $E_{N}$ and $E_{R}$, and we can define an equivalent scalar product in $E_{k}$, by 


$$
\langle\langle U, V\rangle\rangle_{E_{k}}=\Phi\left(P_{N} U, P_{N} V\right)+\Psi\left(P_{R} U, P_{R} V\right) .
$$

where $P_{N}$ and $P_{R}$ are respectively the projections of $E_{k} \rightarrow E_{N}$ and $E_{k} \rightarrow E_{R}$. Rewrite (3.29) as follows

$$
\langle\langle U, V\rangle\rangle_{E_{k}}=\Phi(U, V)+\Psi(U, V) .
$$

We proceed then to show that the subspaces $E_{k_{1}}$ and $E_{k_{2}}$ defined in (3.21), (3.22) are orthogonal with respect to the scalar product (3.29). In fact, it is sufficient to show that $E_{N}$ is orthogonal to $E_{S}$, in turn, this reduces to showing that $\left\langle\left\langle U_{h}^{-}, U_{h}^{+}\right\rangle\right\rangle_{E_{k}}=0$ if $U_{h}^{-} \in E_{N}$ and $U_{h}^{+} \in E_{S}$. Recalling (3.27) and (3.28), we immediately compute that

$$
\begin{aligned}
\left\langle\left\langle U_{j}^{+}, U_{j}^{-}\right\rangle\right\rangle_{E_{k}}=\Phi\left(U_{j}^{+}, U_{j}^{-}\right) \\
=\beta\left(\nabla^{2 m+k} u_{j}, \nabla^{2 m+k} \bar{u}_{j}\right)+2 \beta\left(-\lambda_{j}^{-} \nabla^{-2 m-k} \bar{u}_{j}, \nabla^{2 m} u_{j}\right) \\
+2 \beta\left(-\lambda_{j}^{+} \nabla^{-2 m-k} u_{j}, \nabla^{2 m} \bar{u}_{j}\right)+4\left(-\lambda_{j}^{+} \nabla^{-2 m-k} u_{j},-\lambda_{j}^{-} \nabla^{-2 m-k} u_{j}\right) \\
-4 M\left(\left\|\nabla^{m} u\right\|_{p}^{p}\right)\left(\nabla^{k} \bar{u}_{j}, \nabla^{k} u_{j}\right)+\left(2 \beta^{2}-\beta\right)\left(\nabla^{2 m+k} \bar{u}_{j}, \nabla^{2 m+k} u_{j}\right) \\
=\beta\left\|\nabla^{2 m+k} u_{j}\right\|^{2}-2 \beta\left(\lambda_{j}^{-}+\lambda_{j}^{+}\right)\left\|\nabla^{-k} u_{j}\right\|^{2}+4 \lambda_{j}^{-} \lambda_{j}^{+}\left\|\nabla^{-2 m-k} u_{j}\right\|^{2} \\
-4 M(s)\left\|\nabla^{k} u_{j}\right\|^{2}+\left(2 \beta^{2}-\beta\right)\left\|\nabla^{2 m+k} u_{j}\right\|^{2} \\
=2 \beta^{2} \mu_{j}-2 \beta\left(\lambda_{j}^{-}+\lambda_{j}^{+}\right)+4 \lambda_{j}^{-} \lambda_{j}^{+} \frac{1}{\mu_{j}}-4 M(s) .
\end{aligned}
$$

According to (3.15), we have

$$
\begin{aligned}
& \lambda_{j}^{-}+\lambda_{j}^{+}=\beta \mu_{j} . \\
& \lambda_{j}^{-} \lambda_{j}^{+}=M \mu_{j} .
\end{aligned}
$$

Therefore

$$
\left\langle\left\langle U_{j}^{+}, U_{j}^{-}\right\rangle\right\rangle_{E_{k}}=\Phi\left(U_{j}^{+}, U_{j}^{-}\right)=0 .
$$

step 3 Further, we estimate the Lipschitz constant $l_{F}$ of $F(U)=(0, f(x)-g(u))^{\mathrm{T}}$, according to Lemma 3.3 we can get $g: H_{0}^{2 m}(\Omega) \rightarrow L^{2}(\Omega)$ is uniformly bounded and globally Lipschitz continuous. For $\forall U(u, v)^{\mathrm{T}} \in E_{k}, U_{i}=\left(u_{i}, v_{i}\right)^{\mathrm{T}} \in P_{i} U(i=1,2)$, we have

$$
\begin{aligned}
\|U\|_{E_{k}}^{2} & =\Phi\left(P_{1} U, P_{1} U\right)+\Psi\left(P_{2} U, P_{2} U\right) \\
& \geq\left(\beta^{2} \mu_{j}^{2}-4 M(s)\right)\left\|\nabla^{k} P_{1} u\right\|^{2}+\left(\beta^{2} \lambda_{1}^{m}-4 \mu_{1}\right)\left\|\nabla^{k} P_{2} u\right\|^{2} \\
& \geq\left(\beta^{2} \mu_{j}^{2}-4 M(s)\right)\left\|\nabla^{k} u\right\|^{2} .
\end{aligned}
$$

Given $U=(u, v)^{\mathrm{T}}, V=(\tilde{u}, \tilde{v})^{\mathrm{T}}=(y, z)^{\mathrm{T}} \in E_{k}$, we have

$$
\|F(U)-F(V)\|_{E_{k}}=\|g(u)-g(\tilde{u})\| \leq l\|u-\tilde{u}\| \leq \frac{1}{\sqrt{\beta^{2} \mu_{j}^{2}-4 M(s)}}\|U-V\|_{E_{k}} .
$$

Thus, we have 


$$
l_{F} \leq \frac{1}{\sqrt{\beta^{2} \mu_{j}^{2}-4 M(s)}} .
$$

step 4 Now, we will show the spectral gap condition (3.4) holds.

Since $\Lambda_{1}=\lambda_{N}^{-}, \Lambda_{2}=\lambda_{N+1}^{-}$, then

$$
\Lambda_{2}-\Lambda_{1}=\lambda_{N+1}^{-}-\lambda_{N}^{-}=\frac{\beta}{2}\left(\mu_{N+1}-\mu_{N}\right)+\frac{1}{2}(\sqrt{R(N)}-\sqrt{R(N+1)}) .
$$

where $R(N)=\beta^{2} \mu_{N}^{2}-4 M \mu_{N}^{2}$.

There exists $N_{1} \geq 0$, such that for $\forall N \geq N_{1}$,

$$
\begin{gathered}
R_{1}(N)=1-\sqrt{\frac{\beta^{2}}{\beta^{2} \mu_{j}^{2}-4 M(s)}-\frac{4 M}{\beta^{2} \mu_{j}^{2}-4 M(s)}} \cdot \text { We can get } \\
\quad \sqrt{R(N)}-\sqrt{R(N+1)}+\sqrt{\beta^{2} \mu_{j}^{2}-4 M(s)}\left(\mu_{N+1}-\mu_{N}\right) \\
=\sqrt{\beta^{2} \mu_{j}^{2}-4 M(s)}\left(\mu_{N+1} R_{1}(N+1)-\mu_{N} R_{1}(N)\right),
\end{gathered}
$$

According to assumption (A2), we can easily see that

$$
\lim _{N \rightarrow+\infty}\left(\sqrt{R(N)}-\sqrt{R(N+1)}+\sqrt{\beta^{2} \mu_{j}^{2}-4 M(s)}\left(\mu_{N+1}-\mu_{N}\right)\right)=0,
$$

Then according to (3.18) and (3.37)-(3.40), we have

$$
\Lambda_{2}-\Lambda_{1} \geq \frac{1}{2}\left(\left(\mu_{N+1}-\mu_{N}\right)\left(\beta-\sqrt{\beta^{2} \mu_{j}^{2}-4 M(s)}\right)-1\right) \geq \frac{4 l}{\sqrt{\beta^{2} \mu_{j}^{2}-4 M(s)}} \geq 4 l_{F} \text {. }
$$

The Theorem 3.1 is proved.

Theorem 3.2. Under the conclusion of Theorem 3.1, the problem (1.1)-(1.3) exists a family of inertial manifolds $\mu_{k}$ in $E_{k}$

$$
\mu_{k}=\operatorname{graph}(m):=\left\{\zeta_{k}+\gamma\left(\zeta_{k}\right): \zeta_{k} \in E_{k_{1}}\right\}
$$

where $E_{k_{1}}, E_{k_{2}}$ defined in (3.21)-(3.22), and $\chi: E_{k_{1}} \rightarrow E_{k_{2}}$ is Lipschitz continuous function.

\section{Conflicts of Interest}

The authors declare no conflicts of interest regarding the publication of this paper.

\section{References}

[1] Foias, G., Sell, G.R. and Teman, R. (1985) Varieties inertilles des equations differentielles disspatives. Comptes Rendus de I Académie des Sciences, 301, 139-142.

[2] Guo, C.X. and Mu, C.L. (2007) Exponential Attractors for a Non-Classical Diffusion Equation. Journal of Chongqing University (Natural Science Edition), 30, 87-90.

[3] Wu, J.Z., Zhao, P. and Lin, G.G. (2010) An Inertial Manifold of the Damped Boussiqnesq Equation. Journal of Yunnan University, 32, 310-314.

[4] Wu, J.Z. and Lin, G.G. (2010) An Inertial Manifold of the Two-Dimensional Strongly Damped Boussinesq Equation. Journal of Yunnan University, 32, 119-224.

[5] Yang, M.H. and Sun, C.Y. (2010) Exponential Attractor for the Strongly Damped Wave Equations. Nonlinear Analysis, 11, 913-919. 
https://doi.org/10.1016/j.nonrwa.2009.01.022

[6] Huy, N.T. (2012) Inertial Manifolds for Semi-Linear Parabolic Equations in Admissible Space. Journal of Mathematical Analysis and Applications, 386, 894-909. https://doi.org/10.1016/j.jmaa.2011.08.051

[7] Zhao, B. and Lin, G.G. (2013) Inertial Manifolds for Dual Perturbations of the Cahn-Hilliard Equations. Far East Journal of Applied Mathematics, 77, 113-136.

[8] Zhong, Y.S. and Zhong, C.K. (2012) Exponential Attractors for Semigroups in Banach Spaces. Nonlinear Analysis. Theory, Methods \& Applications, 75, 1799-1809. https://doi.org/10.1016/j.na.2011.09.020

[9] Xu, G.G., Wang, L.B. and Lin, G.G. (2014) Inertial Manifolds for a Class of the Retarded Nonlinear Wave Equations. Mathematica Applicata, 27, 887-891.

[10] Yang, Z. and Liu, Z. (2015) Exponential Attractor for the Kirchhoff Equations with Strong Nonlinear Damping and Supercritical Nonlinear. Applied Mathematics Letters, 46, 127-132. https://doi.org/10.1016/j.aml.2015.02.019

[11] Dai, Z.D. and Ma, D.C. (1998) Nonlinear Wave Equation of Exponential Attractor. Chinese Science Bulletin, 43, 1269-1273. https://doi.org/10.1007/BF02883676

[12] Fan, X.M. and Yang, H. (2010) Exponential Attractor and Its Fractal Dimension for a Second Order Lattice Dynamical System. Journal of Mathematical Analysis and Applications, 367, 350-359. https://doi.org/10.1016/j.jmaa.2009.11.003

[13] Lou, R.J., Lv, P.H. and Lin, G.G. (2016) Exponential Attractors and Inertial Manifolds for a Class of Generalized Nonlinear Kirchhoff-Sine-Gordon Equation. Journal of Advances in Mathematics, 12, 6361-6375. https://doi.org/10.24297/jam.v12i6.3849

[14] Lin, G.G. (2019) Dynamic Properties of Several Kinds of the Kirchhoff Equations. Chongqing University Press, Chongqing.

[15] Eden, A., Folas, C., Nicolanenko, B., et al. (1994) Exponential Attractors for Dissipative Evolution Equations. Research in Applied Mathematics, 37, 57-59.

[16] Lin, G.G. and Yang, L.J. (2021) Global Attractors and Their Dimension Estimates for a Class of Generalized Kirchhoff Equations. Advances in Pure Mathematics, 11, 317-333. https://doi.org/10.4236/apm.2021.114020

[17] Lin, G.G. (2011) Nonlinear Evolution Equation. Yunnan University Press, Kunming. 